\title{
Editorial
}

\section{Diabetic macular edema}

\author{
Benjamin P Hale, MD, Steven J Marks, MD \\ Geisinger Medical Center, Department of Ophthalmology, 100 N Academy Ave, Danville
}

PA, USA 17822

Diabetic retinopathy is a frequent manifestation of long standing diabetes mellitus. According to the World Health Organization diabetes affects nine percent of the adult population worldwide (Diabetes Fact Sheet, 2015). Of all of the common diseases of the eye, diabetic retinopathy has a great impact on the working age population and therefore presents a huge socioeconomic burden in all areas of the world.

As the management of systemic diabetes has improved, so has that for the treatment of diabetic manifestations in the eye such as proliferative diabetic retinopathy, tractional retinal detachment, diabetic macular edema, and neovascular glaucoma. The majority of the recent success in managing diabetic eye disease can be attributed to the use of intravitreal vascular endothelial growth factor inhibitors (anti-VEGF). Improvements have also been seen in surgical management with the introduction of small gauge, high speed vitrectomy cutters. Newer imaging modalities including spectral domain optical coherence tomography (SD OCT) and even more recently, wide-field angiography have also played a part in the successful management of diabetic eye disease. The focus of this editorial is specifically the treatment of diabetic macular edema.

Diabetic macular edema is a leading cause of visual impairment in persons with diabetes (Bhagat et al, 2009). The Arlie House Symposium in 1968 followed by the Diabetic Retinopathy Study group (DRS) and Early Treatment Diabetic Retinopathy Study group (ETDRS) in the 1970s and 1980s defined the protocol for the treatment of patients with diabetic retinopathy and guided treatment of proliferative diabetic retinopathy and clinically significant macular edema with photocoagulation (Goldberg et al, 1987; Early Treatment Diabetic Retinopathy Study research group, 1985; Diabetic Retinopathy Study research group, 1976). We know from these early accounts that patients with clinically significant macular edema benefit from focal laser photocoagulation. In fact, in 1985 the ETDRS Study group published that focal laser treatment increases the chance of visual improvement, decreases the frequency of persistent macular edema, and causes only minor visual field losses. They concluded that all eyes with clinically significant diabetic macular edema should be considered for focal photocoagulation. However, what was considered a success 30 years ago was that treatment with focal laser photocoagulation reduced the risk of moderate visual loss by 50 percent. In other words, focal laser photocoagulation prevents half of the treated patients from losing 3 or more lines of visual acuity. Patients still lost vision and only $15 \%$ saw any significant improvement in visual acuity. (Early Treatment Diabetic Retinopathy Study research group, 1985) Laser photocoagulation in the treatment 
of diabetic macular edema is still utilized. However, the majority of treatments of diabetic macular edema involve anti-VEGF injections.

In 2005, Dr. Philip Rosenfeld used bevacizumab for the treatment of neovascular agerelated macular degeneration (Rosenfeld et al, 2005). This lead to the use of Vascular Endothelial Growth Factor (VEGF) inhibitors in a variety of ocular diseases. VEGF is a well-known pro-angiogenic and vascular permeability molecule (Penn et al, 2008). It was quickly realized that inhibition of VEGF in the eye could be a beneficial and safer alternative to intravitreal steroids for treatment of retinal microvascular diseases such as retinal vein occlusion and diabetic retinopathy. Ranibizumab and aflibercept, two other anti-VEGF medications have since been approved for treating age-related macular degeneration and more recently, both have been found FDA approved for the treatment of patients with diabetic retinopathy and diabetic macular edema.

Ranibizumab $0.3 \mathrm{mg}$ (Lucentis, Genentech) was approved for treating diabetic macular edema in 2012 and for diabetic retinopathy in patients with diabetic macular edema in 2015 (package insert - ranibizumab, 2015). Aflibercept $2 \mathrm{mg}$ (Eylea, Regeneron) was approved for diabetic macular edema in 2014 and diabetic retinopathy in patients with diabetic macular edema in 2015 (package insert - aflibercept, 2015). The so called anti-VEGF era for management of diabetic macular edema has shown improvements in acuity and even improvements in overall retinopathy not seen with prior treatments. This has brought about many interesting clinical questions. For example, how often and for how long do these patients need to be treated with an intravitreal injection? Is there a difference between the three medications in anatomic and functional outcomes? What are the ocular and systemic safety concerns? What is the role of laser now? Can the concomitant use of focal laser improve visual outcomes or decrease the burden of injections? A number of research groups and large clinical trials have been organized to answer some of these questions.

The RISE and RIDE studies were multi-center, randomized studies sponsored by Genentech to explore the efficacy and safety of intravitreal ranibizumab in patients with diabetic macular edema. The Rise and Ride Research Group reported rapid and sustainable improvements in vision, reduced risk of further vision loss, and improvement in macular edema in treated patients. Intravitreal ranibizumab doses of $0.3 \mathrm{mg}$ and $0.5 \mathrm{mg}$ and sham were compared. Statistically significant changes in visual acuity were observed as early as 7 days after the first injection of the $0.3 \mathrm{mg}$ and the $0.5 \mathrm{mg}$ doses of ranibizumab. Most importantly, a significant number of patients randomized to either ranibizumab dose sustained a gain of more than 3 lines $(>15$ ETDRS letters) of visual acuity at 24 months (Nguyen et al, 2012).

VIVID and VISTA were similar, multi-center, randomized studies sponsored by Regeneron to explore the efficacy and safety of intravitreal aflibercept in patients with diabetic macular edema. Similar visual acuity outcomes were found, with a few important differences from the Rise and Ride studies. In Vivid and Vista there was a direct comparison of VEGF blockade to laser alone for diabetic macular edema showing a clear benefit of anti-VEGF over focal laser. In Rise and Ride all arms were eligible for laser treatment. Also, aflibercept has the advantage of a more convenient 
dosing regimen of every 8 weeks (after 5 initial monthly loading injections). Patients treated with aflibercept showed improvements in visual acuity, decreased severe vision loss and improvement in overall diabetic retinopathy similar to other anti-VEGF medications (Korobelnik , 2014).

Even more recently and of great interest was the DRCR's protocol T Study, which was published in the New England Journal of Medicine in March 2015 (Wells et al, 2015). They set out to answer the question, "Which anti-VEGF agent is better?". DRCR Protocol $\mathrm{T}$ became the first head to head study comparing visual acuity outcomes at one year in patients with diabetic macular edema treated with bevacizumab, ranibizumab, and aflibercept. What the DRCR found was that all three anti VEGF agents improved patient's visual acuity from baseline. Overall patients receiving aflibercept had a greater improvement in visual acuity than those treated with bevacizumab and ranibizumab. Patients receiving aflibercept showed a 13.3 visual-acuity letter score improvement from baseline at one year versus 9.7 letters with bevacizumab and 11.2 letters with ranibizumab $(\mathrm{P}<0.001$ for aflibercept vs. bevacizumab and $\mathrm{P}=0.03$ for aflibercept vs ranibizumab). Interestingly, subgroup analysis showed that patients with good baseline visual acuity of $20 / 32$ to $20 / 40$ had no statistically significant difference in visual acuity outcomes between anti-VEGF agents. Those with worse baseline visual acuity of less than 20/40, did better on aflibercept.

There are always going to be more questions than answers and the challenge of unique clinical cases. It is not always clear cut which patients are going to need treatment or benefit from treatment of their diabetic macular edema. Factors such as coexisting macular ischemia, chronicity of macular edema, and compliance with follow up, make decisions on treatment challenging. Even when it is clear which patients will benefit from treatment, there are multiple factors to consider in a real world situation, and the choice of anti-VEGF agents still vary among specialists. The results of this multicenter, randomized study may offer some guidance, at least to efficacy, when choosing an anti-VEGF agent.

It is evident that patients treated with frequent anti-VEGF medications such as bevacizumab, ranibizumab, and aflibercept show improved visual and anatomic outcomes over those treated with focal laser or observation. The improvements in acuity are greater and quicker than focal laser alone and local side effects such as cataract and elevated intraocular pressure are less than with intravitreal steroids. We are now able to offer patients a chance at improvement in short and long term visual acuity, rather than a discussion on preventing further vision loss. Frequent treatment with anti-VEGF also shows a reduction in the rate of progression of retinopathy to more severe, proliferative stages and in some patients an improvement in background retinopathy. This theoretically decreases the need for panretinal photocoagulation or vitrectomy. The exact number of patients to benefit from this observation and the sustainability of these improvements after cessation of anti-VEGF therapy is still to be determined (Nguyen et al, 2012), and clinical judgment is still necessary when caring for these complex patients. There is a wide variety of presentations of diabetic macular edema and many patients may have compounding issues such as coexisting 
macular ischemia, proliferative retinopathy, tractional retinal detachment, and systemic poorly controlled hypertension, blood sugar, or fluid status which make management challenging. Many specialists may still advocate focal laser for focal diabetic macular edema with a few associated microaneurysms outside of the fovea and some have decided to abandon laser all together.

As more expensive treatments evolve, the looming issue becomes the treatment of patients that have no access to better treatment due to finances or availability. The treatment burden of anti-VEGF is highest in year one and decreases over time, but certainly for patients living in remote villages in Nepal, regular expensive injections might be impossible. The DRCR showed a sustained visual benefit for patients receiving a mean number of 9 injections of ranibizumab over year one, 3 injections over year two, and 2 injections over year three when focal laser was deferred. When prompt focal laser was performed patients needed only one less injection per year (Elman et al, 2011). This treatment burden is huge in certain areas of the world without health care insurance or nearby clinics for treatment. In this setting, laser photocoagulation still could play a major role.

Expect to see more discussions on steroid implants, wide field imaging, and peripheral laser treatments as dual therapy for the treatment of diabetic macular edema in the next few years. Diabetic retinopathy and diabetic macular edema is a multifactorial disease and thinking of treating it solely with anti-VEGF may be simplifying the disease process too much. Intravitreal steroids such as triamcinolone, Ozurdex (dexamethasone intravitreal implant, Allergan), and Iluvien (fluocinolone acetonide, Alimera Sciences) have endothelial growth factor and anti-inflammatory properties that, in the right patient, can be very beneficial both in visual acuity and anatomical outcomes and in decreasing treatment burden of every 4 or every 8 week injections (package insert - Ozurdex, 2014; package insert - Iluvien, 2014).

The manifestations of diabetes mellitus in the eye can be devastating. The old saying, "an ounce of prevention is worth a pound of cure," applies here and patients should be educated early in the course of their disease about the effects of diabetes on the eye. When diabetic retinopathy and diabetic macular edema do develop, patients should be treated regularly to give them the best chance at a good short term and long term visual outcome.

\section{References}

Bhagat N, Grigorian RA, Tutela A, Zarbin MA (2009). Diabetic macular edema: Pathogenesis and treatment. Survey of Ophthalmology; 54(1): 1-32.

Diabetes Fact Sheet, 2015. World Health Organization. [online] (updated January 2015) Available at: <http://www.who.int/mediacentre/factsheets/fs312/en/> [Accessed online 25 June 2015].

Diabetic Retinopathy Study Research Group (1976). Preliminary report on effects of photocoagulation therapy. American Journal of Ophthalmology; 81: 383. 
Early Treatment Diabetic Retinopathy Study research group. (1985). Photocoagulation for diabetic macular edema. Early Treatment Diabetic Retinopathy Study report number 1. Archives of Ophthalmology; 103:1796-806.

Elman MJ, Bressler NM, Qin H, et al (2011). Expanded 2-year follow-up of ranibizumab plus prompt or deferred laser or triamcinolone plus prompt laser for diabetic macular edema. Ophthalmology; 118(4): 609-14.

Goldberg MF, Jampol LM. (1987). Knowledge of diabetic retinopathy before and 18 years after the Airlie House Symposium on Treatment of Diabetic Retinopathy. Ophthalmology; 94(7): 741-746.

Korobelnik JF, Do DV, Schmidt-Erfurth U (2014). Aflibercept for Diabetic Macular Edema. Ophthalmology; 121(11):2247-2254.

Nguyen QD, Brown DM, Marcus DM et al (2012). Ranibizumab for Diabetic Macular Edema: Results from 2 Phase III Randomized Trials: RISE and RIDE. Ophthalmology; 119: 789-801.

Package insert, 2014. Allergan. [online] Available at: http://www.allergan.com/assets/pdf/ ozurdex_pi.pdf [Accessed online 6 August 2015].

Package insert, 2014. Alimera Sciences. [online] Available at: http://www.alimerasciences. com/wp-content/uploads/2014/09/iluvien-prescribing-information.pdf [Accessed online 6 August 2015].

ackage insert, 2015. Genentech. [online] Available at: http://www.gene.com/download/pdf/ lucentis_prescribing.pdf [Accessed online 6 August 2015].

Package insert, 2015. Regeneron. [online] Available at: http://www.regeneron.com/Eylea/ eylea-fpi.pdf [Accessed online 6 August 2015].

Penn JS, Madan A, Caldwell RB, Bartolie M, Caldwell RW, Hartnett ME (2008). Vascular Endothelial Growth Factor in Eye Disease. Progress in Retinal and Eye Research; 27 (4):331-71.

Rosenfeld PJ, Moshfeghi AA, Puliafito CA (2005). Optical Coherence Tomography Findings after an Intravitreal Injection of bevacizumab (Avastin) for Neovascular Age-related Macular Degeneration. Ophthalmic Surgery, Lasers \& Imaging: the Official Journal of the International Society of Imaging in the Eye; 36(4): 331-335.

Wells JA, Glassman AR, Ayala AR, et al (2015). Diabetic Retinopathy Clinical Research Network: Comparative Effectiveness Randomized Clinical Trial of Aflibercept, Bevacizumab, or Ranibizumab for Diabetic Macular Edema. New England Journal of Medicine; 372:1193-1203. 